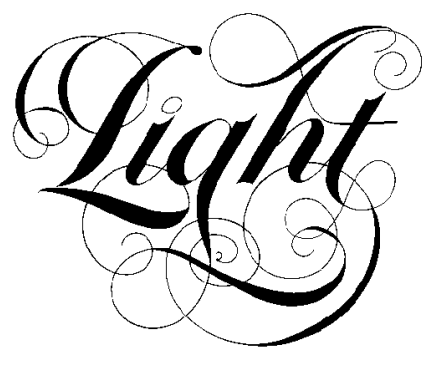

\title{
Indium Tin Oxide Overlayered Waveguides for Sensor Applications
}

B.J.Luff, J.S.Wilkinson and $*$ G.Perrone

*Dipartimento di Elettronica, Politecnico di Torino, Torino, Italy

\begin{abstract}
The use of indium tin oxide (ITO) thin films as electrodes for integrated optical electrochemical sensor devices is discussed. The effect of various thicknesses of ITO overlayers exhibiting low resistivity and high transparency on potassium ion-exchanged waveguides fabricated in glass substrates is investigated over the wavelength range $500-900 \mathrm{~nm}$. ITO overlayers are formed by reactive thermal evaporation in oxygen, followed by annealing in air to a maximum temperature of $320^{\circ} \mathrm{C}$. With air as superstrate, losses in the waveguides were found to increase dramatically above $30 \mathrm{~nm}$ ITO thickness for TE polarization, and above 50nm thickness for TM. Losses were increased over the whole wavelength range for a superstrate index close to that of water. A one-dimensional multilayer waveguide model is used in the interpretation of the experimental results.
\end{abstract}

\section{Introduction}

Integrated optical chemical and biochemical sensors are currently the subject of much research effort. ${ }^{1-3}$ In such sensors, the high sensitivity of optically-based transduction methods is combined with the compact and rugged format of an integrated optical device. Although some applications would benefit from cheap 'single-shot' devices, perhaps capable of detecting a single substance or a class of closely related substances, it is highly desirable both to extend the number of different substances that can be tested simultaneously with a single sensing element and to make such a sensor regenerable so that it may be used for many consecutive measurements. To this end, electrochemical control over interactions taking place on waveguides can be a considerable advantage. Several chemical sensors featuring electrochemical control of reactions on glass waveguides have been demonstrated. ${ }^{4-8}$ Suitable electrode overlayers for waveguides need to have the correct combination of electrical and optical properties: generally, low resistivities are required along with high transparency over the wavelength range of operation. It should also be possible to pattern the material using standard microlithographic techniques in order to define specific regions on a device. Further, sensing applications will often require the stable attachment of chemical species to the electrode surface, and the material must be amenable to surface modification to enable this attachment.

We can identify three main types of waveguide sensor to which electrochemical control using indium tin oxide (ITO) electrodes may be applied: absorption sensors, ${ }^{5,9}$ where an absorbing medium in the superstrate produces a direct change in transmitted light intensity through the waveguide; refractive index sensors, ${ }^{3,10}$ where the propagation coefficient of the waveguide mode is altered due to refractive index changes in the superstrate; and fluorescence or chemiluminescence-based sensors, ${ }^{6,11,12}$ in which light emission from sources in the evanescent region is excited and/or collected by the waveguide. Specific sensor devices usually require that chemical or biochemical species be immobilized on the waveguide surface. Suitable attachment techniques for ITO have been developed. ${ }^{6,13}$ In this paper we investigate the use of thin ITO over layers on waveguides for electrochemical applications over the wavelength range 500-900nm. Waveguides fabricated by ion-exchange were employed because this technique is finding increasing importance as an inexpensive technology for the production of integrated optical devices. ${ }^{14}$ 
The ITO films are shown to have the required properties for use as waveguide electrode over layers, and experimental and theoretical results are presented that will be of use in the design of waveguide sensors utilizing this material. In addition to measurements made using air as a superstrate, a superstrate index near that of water is also considered, as many electrochemical reactions of interest in sensing applications take place in an aqueous environment.

\section{Theoretical model}

Figure 1 shows parameters for a 1D multilayer waveguide. The index variation of the waveguide is assumed to be Gaussian (Subsection 3.A) of the form
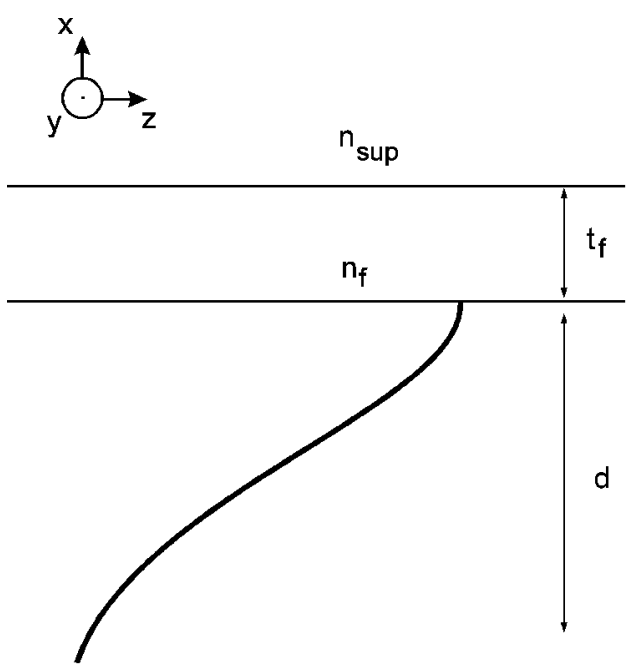

Fig. 1 One-dimensional multilayer waveguide parameters: $n_{\text {sup }}$, superstrate index; $n_{f}$, film index; $t_{f}$, film thickness; $d$, diffusion depth.

$$
n(x)=\Delta n \exp \left|-\frac{x^{-}}{x^{2}}\right|
$$

The effective indices of waveguide modes of the multilayer structure have been computed by solving the equivalent transmission line problem obtained after a staircase approximation is used to discretize the index profile. ${ }^{15}$

Once the value of the propagation constant has been found, the corresponding field profile can be determined. For TE polarization, the modal solution is of the form $E_{y}(x, z)=\mathrm{E}_{\mathrm{yo}}(x) \exp (-i \beta z)$, where $\beta$ is the complex propagation constant and $E_{y o}(x)$ is the field profile. Writing $\beta$ as $\beta^{\prime}-\beta^{\prime \prime} i$, we have

$$
E_{y}=E_{y o} \exp \left(-\beta^{\prime \prime} z\right) \exp \left(-i \beta^{\prime} z\right)
$$

i.e., the mode decays exponentially with $z$ and the rate of decay depends on the imaginary part of the propagation constant. The effective index $n_{\text {eff }}=n_{\text {eff }}{ }^{\prime}-n_{\text {eff }}{ }^{\prime \prime} i$ is related to the propagation constant by $\beta=2 \pi n_{\text {eff }} / \lambda$, where $\lambda$ is the wavelength. The attenuation $\alpha$ in decibels per centimeter is then given by

where $\lambda$ is in $\mathrm{nm}$.

$$
\alpha=\left(4.34 \times 10^{7}\right) \frac{4 \pi n_{e f f}{ }^{\prime \prime}}{\lambda}
$$




\section{Sample preparation}

\section{A. Waveguide fabrication}

Waveguides were fabricated in $1 \mathrm{~mm}$ thick soda-lime glass microscope slides (ROWI) by immersion in a $\mathrm{KNO}_{3}$ melt at $390^{\circ} \mathrm{C}$ for 40 $\mathrm{m}$ i n u t e s. A photolithographically patterned aluminium mask was used to define the waveguide channels; the channel width was $3 \mu \mathrm{m}$.

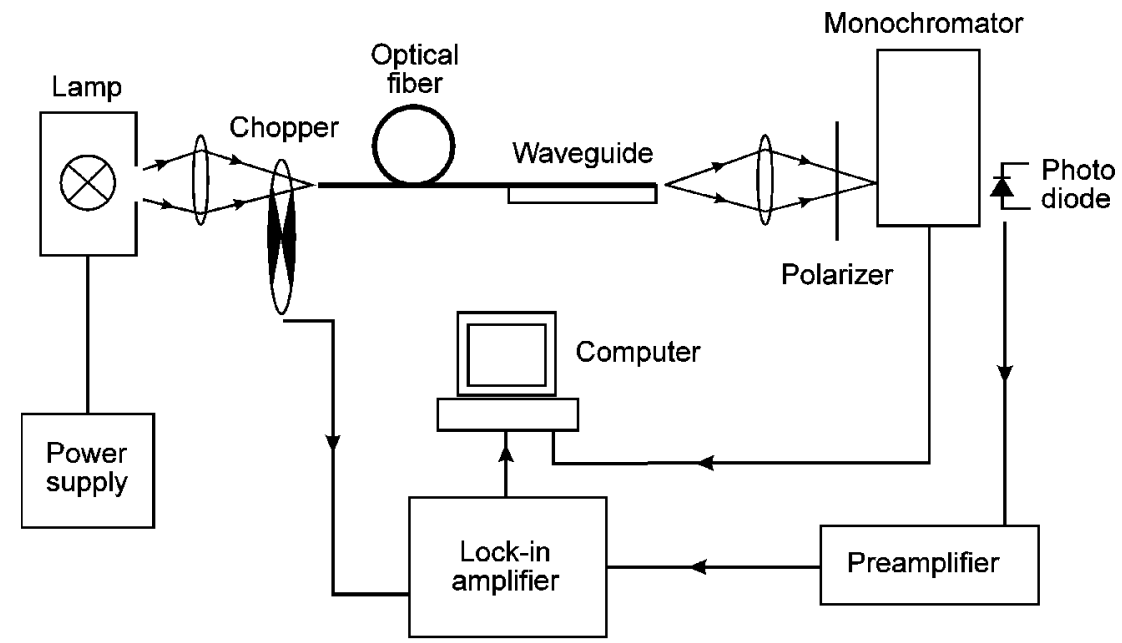

Fig. 2 Waveguide attenuation measurement apparatus.

The resulting waveguides were single-moded at wavelengths above $\sim 550 \mathrm{~nm}$ with cutoffs at around $800 \mathrm{~nm}$ (Section 5). Fabrication of planar waveguides by $\mathrm{K}^{+}$ion-exchange in the microscope slides used for this work produces a diffused profile with a maximum index change at the surface $(\Delta \mathrm{n})$ of 0.01 for TM polarization and 0.008 for TE with a Gaussian diffusion profile, as determined by mode index measurements ${ }^{16}$; the expected diffusion depth is approximately $2 \mu \mathrm{m} .{ }^{17}$ However, the depths of channel and planar waveguides diffused for the same duration can be significantly different. ${ }^{18}$ The birefringence exhibited is a feature of waveguides fabricated in glass by $\mathrm{K}^{+}-\mathrm{Na}^{+}$ion-exchange. ${ }^{19}$ The ITO annealing treatment described in Section 3.B is expected to reduce the index change at the surface of an ion-exchanged waveguide and deepen the index profile, giving the waveguide a larger cross section, reducing the field intensity at the waveguide surface for light propagating in the waveguide. ${ }^{20}$

A single $50 \times 50 \mathrm{~mm}$ ion-exchanged sample was cut into six samples of dimensions $20 \times 15 \mathrm{~mm}$ and the waveguides end polished for use in measurements.

\section{B. ITO films}

ITO films (indium oxide doped with tin) are used extensively in displays, solar cells and a variety of photosensitive devices. Their use as electrodes in waveguide modulators has been described. ${ }^{21}$ High quality films have been prepared by several methods including vacuum evaporation, ${ }^{22,23} \mathrm{rf}$ sputtering,${ }^{24} \mathrm{dc}$ sputtering ${ }^{25}$ and chemical vapour deposition (CVD). ${ }^{26}$ The optical and electronic properties of films vary widely depending on deposition method, substrate temperature, whether the deposition is reactive (i.e., carried out under a partial pressure of reactive gas) and on the post-treatment of the films e.g., annealing. Generally, sheet resistance values of high quality films are quoted from around $10 \Omega / \mathrm{sq}$

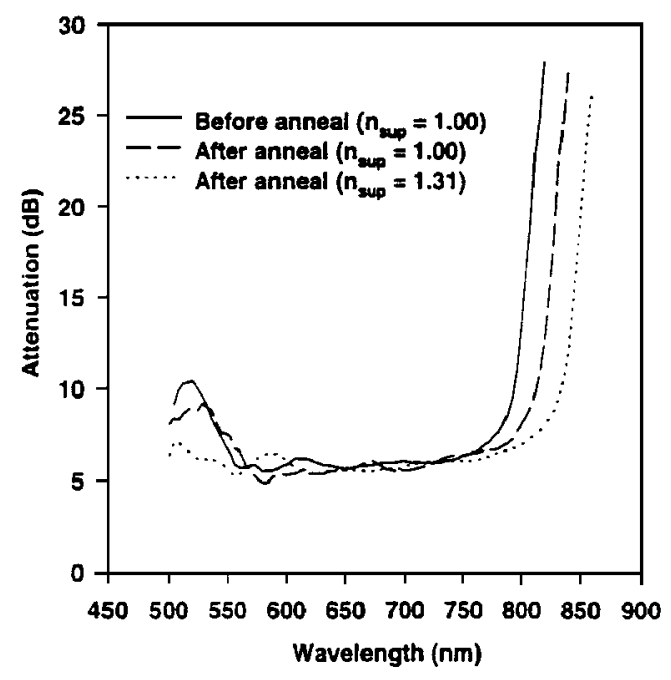

Fig. 3 Effect of anneal on a non-ITO coated waveguide (TE polarization). 
to several $\mathrm{k} \Omega / \mathrm{sq}$, and transmissions in the visible range from $80 \%$ to better than $90 \%$. Thicknesses of films vary considerably from application to application, from less than $20 \mathrm{~nm}$ to nearly one micron.

For electrochemical applications on waveguides sheet resistances of $<1 \mathrm{k} \Omega / \mathrm{sq}$ are required ${ }^{4}$ and the films should exhibit high transmission in the wavelength range of interest. For this work, ITO material (91 mol\% $\mathrm{In}_{2} \mathrm{O}_{3}-9$ mol\% $\mathrm{SnO}_{2}, 99.997 \%$ purity) obtained from Cerac Inc. (Milwaukee, USA) was evaporated in an Edwards E306A coater from an integrated tungsten/alumina crucible at an $\mathrm{O}_{2}$ partial pressure of $5 \times 10^{-4} \mathrm{mbar}$. The pressure in the chamber before introducing the oxygen was $2 \times 10^{-6}$ mbar or less. Film thickness and rate of deposition were monitored using a quartz crystal monitor, which was first calibrated by measuring the thicknesses of deposited films (after annealing) using a Tencor Alphastep surface profiler. Steps for profiling were etched with a $37 \% \mathrm{HCl}$ solution ${ }^{27}$ after defining an edge using photoresist; this process also demonstrates the suitability of the films for photolithographic patterning.

Sheet resistances $R$ of films were measured using a conventional four point probe method; the sheet resistance is related to the resistivity by $R=\rho / t_{f}$, where $\rho$ is the resistivity and $t_{f}$ is the film thickness. Directly after evaporation, the ITO films exhibit poor transmission in the visible region $(<60 \%)$ and high sheet resistance $(>10 \mathrm{k} \Omega / \mathrm{sq})$. To improve the quality of the films, the samples were placed in an oven at room temperature and the temperature ramped to a maximum of $320^{\circ} \mathrm{C}$ over 90 minutes; the oven was then switched off and the samples left in the oven for 5 hours to cool to room temperature. Following this treatment, the sheet resistance was found to be reduced significantly to between 150 and $900 \Omega / s q$ and the transparency increased (Section 5). Good electrochemical stability for ITO thin film electrodes has been demonstrated. ${ }^{28}$

\section{Waveguide attenuation measurements}

Waveguide attenuation measurements were made using the apparatus shown in Figure 2. Chopped light from a tungsten-halogen source was coupled into a single mode optical fiber. The fiber was butt-coupled to the waveguides and the output from the guides collected by an objective lens and focused onto the monochromator slit. The intensity at the output slit was measured using a Si photodetector and lock-in amplification. The polarizer was included to select TE or TM polarization. Spectra were taken initially for both polarizations with no sample in place and with the fiber close to the output objective (so that all light from the fiber was collected). Spectra taken with the samples in place were then ratioed to these reference spectra to determine the total attenuation, which includes the fiber-waveguide coupling loss. The wavelength range of the measurements was 500-900 nm.

\section{Results and discussion}

One of the six samples was subjected to the ITO annealing treatment without having an ITO film evaporated onto it, in order to determine the effect of annealing on the waveguide losses. Figure 3 , attenuation spectra for TE polarized light, shows that the anneal produces a small cutoff shift to longer wavelengths due to a redistribution of the exchanged ions and a corresponding broadening of the refractive index profile, as mentioned in Section 3.A, but has no significant effect on the losses of the waveguides. Also shown in Figure 3 is the additional wavelength shift 


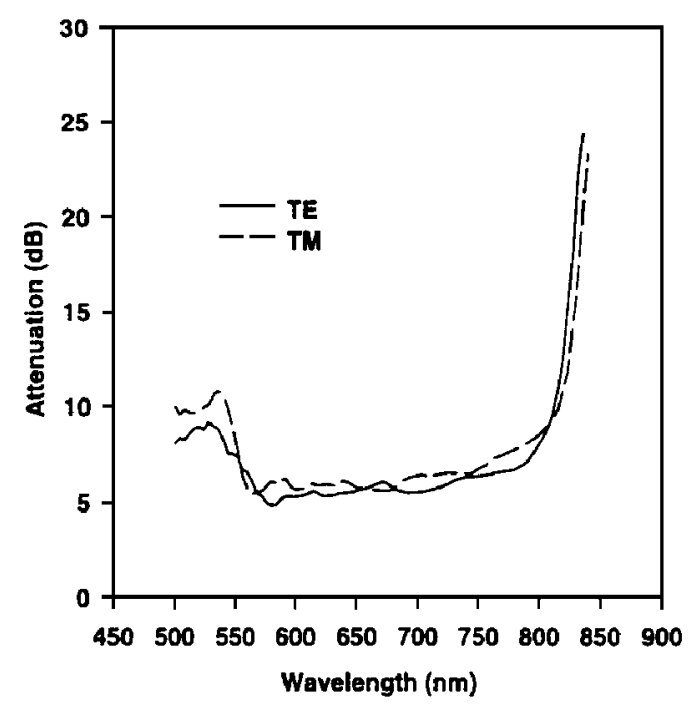

Fig. 4 Comparison of TE and TM spectra for the annealed, uncoated sample $\left(\mathbf{n}_{\text {sup }}=\right.$ 1.00).

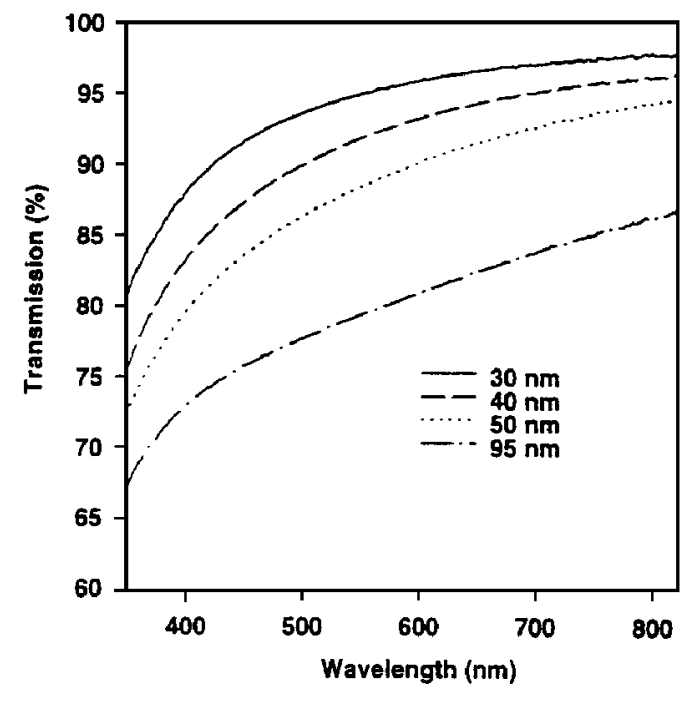

Fig. 5 Bulk transmission spectra of the ITO films

in the cutoff introduced by increasing the superstrate index to a value approaching that of aqueous solutions. The refractive index of distilled water is close to 1.333 ; in this work, for convenience, the superstrate index was increased to $n_{\text {sup }}=1.31$ by evaporation of a $1 \mu \mathrm{m}$ thick film of Teflon $\mathrm{AF} 1600,{ }^{29}$ a highly transparent fluoropolymer material that causes negligible additional signal absorption. Similar spectra were seen for TM polarization; Figure 4 compares the spectra from the annealed sample for TE and TM polarizations. The apparent increase in attenuation below $550 \mathrm{~nm}$ seen in the spectra is due to the waveguides becoming multi-moded. The loss of approximately $5 \mathrm{~dB}$ in the monomode operating region is dominated by the fiber coupling loss and Fresnel losses.

Table 1. ITO film sheet resistance $R$ where $t_{f}$ is film thickness

\begin{tabular}{|c|c|}
\hline $\mathrm{t}_{\mathrm{f}} \pm 5(\mathrm{~nm})$ & $\mathrm{R} \pm 30(\Omega / \mathrm{sq})$ \\
\hline 30 & 850 \\
40 & 232 \\
50 & 225 \\
96 & 230 \\
\hline
\end{tabular}

Table 2. ITO film transmissions at $633 \mathrm{~nm}$, measured without a reference sample ${ }^{\mathrm{a}}$

\begin{tabular}{|c|c|c|}
\hline $\begin{array}{c}\text { Film thickness } \\
\mathrm{t}_{\mathrm{f}} \pm 5(\mathrm{~nm})\end{array}$ & $\begin{array}{c}\text { Measured transmission } \\
\mathrm{T}_{\text {meas }} \pm 0.5(\%)\end{array}$ & $\begin{array}{c}\text { Calculated transmission } \\
\mathrm{T}_{\text {calc }}(\%)\end{array}$ \\
\hline 30 & 88.6 & 87.5 \\
40 & 85.6 & 85.7 \\
50 & 83.5 & 83.9 \\
95 & 78.2 & 79.7 \\
\hline
\end{tabular}

The attenuation spectra of waveguides on the remaining five samples were measured for both polarizations, and four of these samples were then coated with ITO over layers with thicknesses 


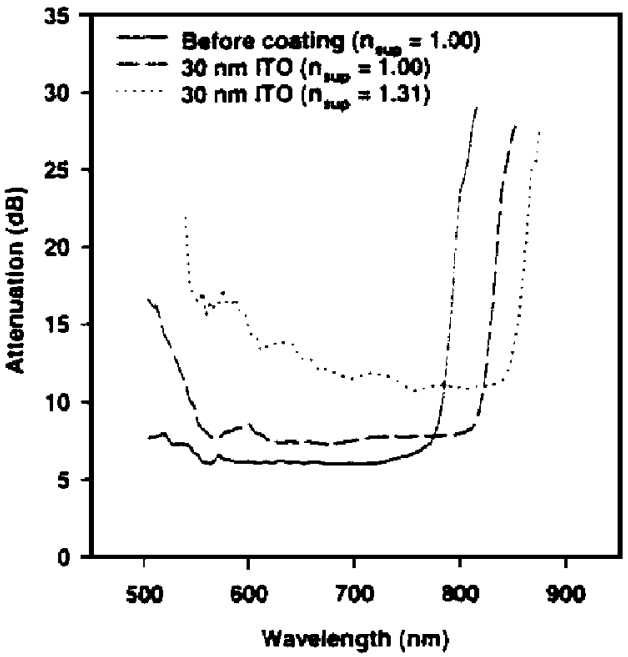

(a)

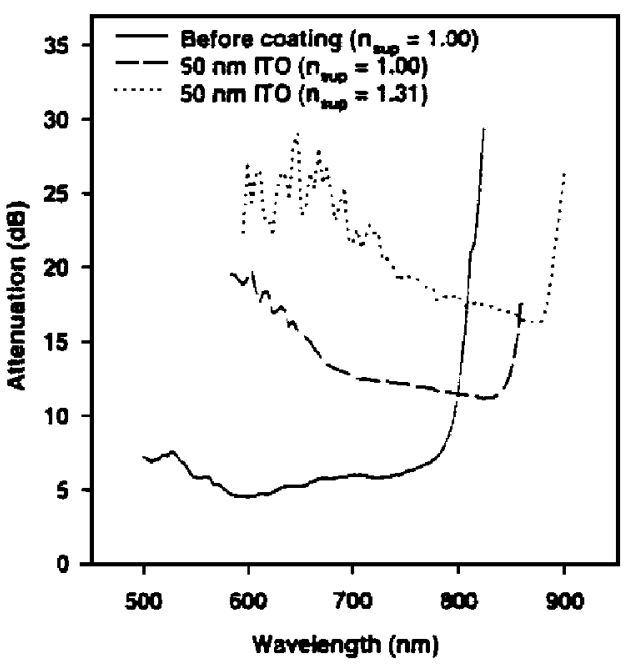

(b)

Fig. 6 Effect of (a) 30nm and (b) 50nm ITO film on waveguide attenuation spectra, measured for $n_{\text {sup }}=1.00$ and 1.31

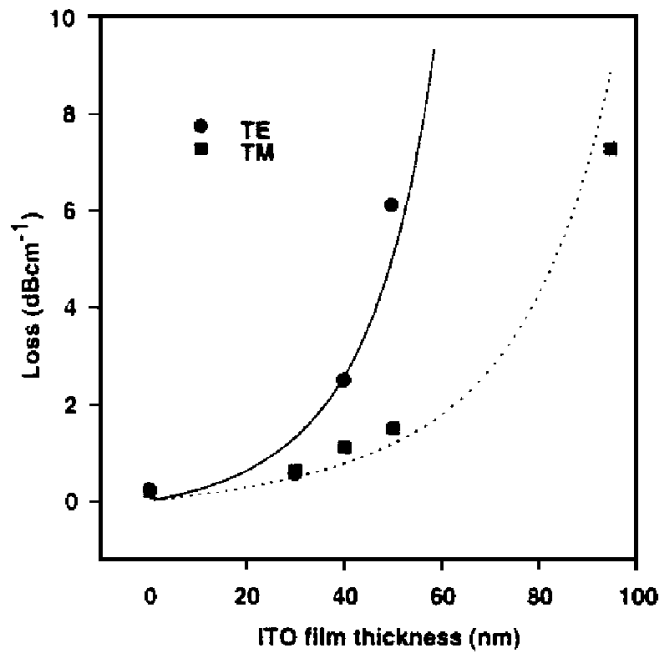

(a)

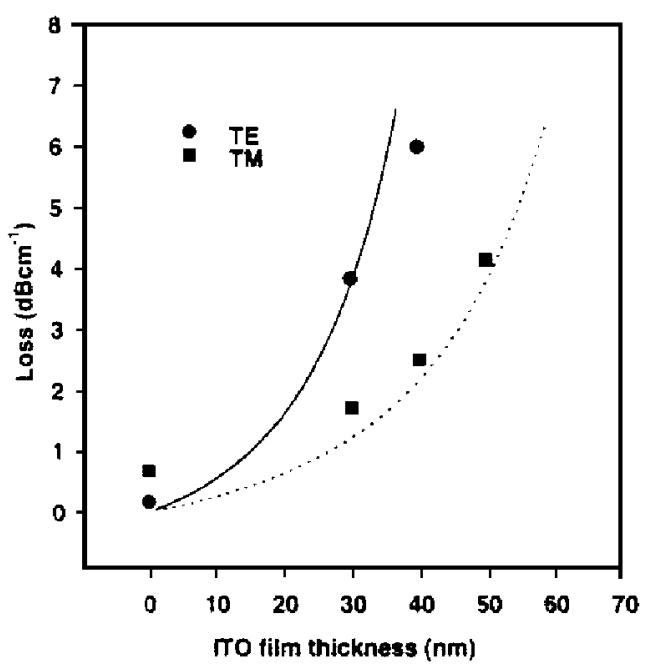

(b)

Fig. 7 Attenuation at $633 \mathrm{~nm}$ due to ITO overlayers for (a) $\mathbf{n}_{\text {sup }}=\mathbf{1 . 0 0}$ and (b) $\mathbf{n}_{\text {sup }}=$ 1.31. Individual points show measured values; solid and dotted curves show the modeled attenuation for TE and TM polarizations, respectively.

30, 40, 50 and $95 \mathrm{~nm}( \pm 5 \mathrm{~nm})$ and annealed as described in Subsection 3.B. Figure 5 shows measurements of bulk transmission spectra taken normal to the films over the wavelength range 360-820nm; the measurements were made using a Perkin-Elmer dual-beam spectrophotometer with an uncoated microscope slide as a reference. The transmission decreases monotonically with thickness over the whole spectral range of the measurement. Table 1 gives the values obtained for the sheet resistances of the films. There is a sharp drop in sheet resistance from $850 \Omega / \mathrm{sq}$ for the $30 \mathrm{~nm}$ film to $232 \Omega / \mathrm{sq}$ for the $40 \mathrm{~nm}$ film, but the sheet resistance then does not change significantly for the 50 and $95 \mathrm{~nm}$ films. This is likely to be due to normal fabrication tolerance; over a large number of samples the average sheet resistance would be expected to decrease with increasing film thickness.

The ITO refractive index is expressed as $n_{f}=n_{f}{ }^{\prime}-n_{f}{ }^{\prime \prime} i$. The complex index of the $95 \mathrm{~nm}$ film was 
measured using variable angle ellipsometry at $633 \mathrm{~nm}$; the real part of the ITO index $n_{f}{ }^{\prime}$ was determined as 1.78 and the imaginary part $n_{f}^{\prime \prime}$ as 0.03 . These values are comparable with published results. ${ }^{30-32}$ In order to gain further confidence in these values for the ITO index, transmissions at $633 \mathrm{~nm}$ were measured without a reference sample for each film thickness and compared with transmission values calculated using these index values and the measured film thicknesses; a good agreement is found, as shown in Table 2. For these calculations, a matrix method for a single absorbing film on a transparent substrate was used, ${ }^{33}$ so that multiple reflections within the ITO film are taken into account; a loss of $4 \%$ on exiting the substrate was assumed.

The attenuation spectra for the waveguides measured when uncoated were then measured again with the ITO coatings for air and Teflon AF superstrates. Figures 6(a) and (b) show examples of waveguide attenuation spectra taken before and after the addition of the $30 \mathrm{~nm}$ and $50 \mathrm{~nm}$ ITO films respectively for TE polarization; as expected, the presence of the ITO films increases the waveguide losses. In addition, an increase in cutoff wavelength is observed. The figures also show that increasing the superstrate index significantly increases the attenuation in the ITO coated waveguides and introduces a further shift in cutoff. The increased attenuation is a result of increased evanescent field interaction with the ITO film due to the waveguide system becoming more symmetric; similar field enhancements due to increasing symmetry can generally be achieved by lowering the substrate index to make it closer to that of the superstrate or by increasing the index of the waveguide.

The attenuation in $\mathrm{dBcm}^{-1}$ due to each thickness of ITO film was calculated for both TE and TM polarization and for $n_{\text {sup }}=1.00$ and 1.31 by subtracting the attenuation measured before coating from that measured after, and then dividing by the length of the samples $(2 \mathrm{~cm})$. Figures 7 (a) and (b) show the results of the measurements at $633 \mathrm{~nm}$. For TE polarization, no signal could be measured for the $95 \mathrm{~nm}$ film; this is partly due to poor spectrometer grating efficiency for this polarization. The figures also show the losses calculated using the 1D model with a Gaussian diffusion profile described in Section 2, which is seen to describe the measured behaviour well. For the purposes of the model, the ITO film is assumed to be the only lossy component of the multilayer waveguide. The index of the glass substrate is taken as $n_{\text {sub }}=1.512$. Values for the 1D guide surface index change $\Delta n$ and diffusion depth $d$ representative of the actual 2D channel waveguide are determined by fitting the measured points for $n_{\text {sup }}=1.00$ to the model by minimization of the function

$$
f(\Delta n, d)=g(V) \sum_{i}\left|\frac{L_{c}(i)^{2}}{L_{m}(i)^{2}}-1\right|,
$$

where $L_{c}(i)$ and $\mathrm{L}_{\mathrm{m}}$ (i) are the calculated and measured losses, respectively, for the point $(i)$ and the sum over the points $(i)$ includes those for both TE and TM polarizations; $g(V)$ is a penalty function that forces the retrieval of $\Delta n$ and $(d)$ corresponding to a monomode index profile

$$
g(V)=\left\{\begin{array}{cc}
1 & \mathrm{~V}<\mathrm{V}_{\mathrm{c}} \\
10^{3} \mathrm{~V}>\mathrm{V}_{\mathrm{c}}
\end{array}\right.
$$

where the normalized diffusion depth is defined as 


$$
V=\frac{2 \pi}{\lambda} d\left[\left(n_{\mathrm{sub}}+\Delta n\right)^{2}-n_{\mathrm{sub}}^{2}\right]^{1 / 2}
$$

and $V_{c}$ is the cutoff value of the normalized diffusion depth for the second mode. For the purposes of the model we ignore any birefringence effects. The values determined from the fitting procedure were $\Delta n=0.0016$ and $d=3.96 \mu \mathrm{m}$. In Fig.7(b) the same waveguide parameters as determined for $n_{\text {sup }}=1.00$ are used in the model with $n_{\text {sup }}=1.31$. The model is seen to predict the measured behaviour for the higher superstrate index well, showing significant increases in attenuation for both TE and TM polarizations due to the increase in waveguide symmetry as discussed above.

The value of $\Delta n$ appears rather low for potassium ion-exchanged waveguides in soda-lime glass (Section 3.A). However, the annealing of the samples described in Section 3.B reduces the peak potassium concentration and hence the surface index change; further, the value of the equivalent index change used as a parameter in a 1D waveguide model is expected to be significantly lower than that of an actual channel waveguide. The diffusion depth (expected to be approximately $2 \mu \mathrm{m}$ for the unannealed waveguides) will also be increased by the annealing treatment, consistent with the value of $3.96 \mu \mathrm{m}$ determined by fitting the data. Confidence in the parameters used in the model is strengthened by the observation that modelled values for the attenuation as a function of ITO thickness are in good agreement with experimental results for both air and Teflon AF superstrates.

From Figure 7, where the ITO film covers the whole guide length of $2 \mathrm{~cm}$, acceptable losses occur only for ITO film thicknesses less than around 40nm for TE polarized light and 50nm for TM, with $n_{\text {sup }}=1.00$. In the case of a superstrate index near that of water, losses are somewhat higher although still acceptable for the thinner films. In many applications the ITO film will not be required to cover the whole waveguide length (usually of several centimetres) and photolithographically patterned lengths of a few millimetres may be sufficient, significantly reducing losses due to the overlayer. The attenuation is generally found to be higher for shorter wavelengths for all film thicknesses, especially for $n_{\text {sup }}=1.31$, although this trend is less marked for TM polarization.

In order to assess the sensitivity of ITO overlayered waveguides in a typical sensor configuration, we consider their application in an integrated optical Mach-Zehnder interferometer device, ${ }^{3}$ where the ITO overlayered waveguide forms the 'sensing' arm. The phase change produced in such a device due to a change in the real part of the effective index of the sensing arm $\left(\Delta n_{\text {eff }}{ }^{\prime}\right)$ is given by $\Delta n_{\text {eff }}{ }^{\prime} k_{0} L$, where $k_{0}$ is the wave vector and $L$ is the interaction length. For a $30 \mathrm{~nm}$ thick ITO film extending over an interaction length of $15 \mathrm{~mm}$, (taking $\lambda$ as $632.8 \mathrm{~nm}$, TE polarization, and assuming a water superstrate, $n_{\text {sup }}=1.333$ ), the theoretical loss introduced by the film is $6.6 \mathrm{~dB}$, which will not have a significant effect on signal detectability. We take $5 \times 10^{-4} \pi$ radians as the minimum detectable phase change in a typical measurement setup (cf. Ref. 34). Application of the 1D multilayer model shows that this minimum detectable phase shift is produced for a change in bulk superstrate index of $4 \times 10^{-5}$. Thus, ITO overlayered waveguides offer reasonable sensitivity for practical sensor devices. It is of interest to compare this sensitivity with the sensitivity of an annealed waveguide without the high-index ITO layer above. We find for this case that the minimum detectable change in superstrate refractive index is $8 \times 10^{-5}$; thus the presence of the thin high-index ITO overlayer increases the sensitivity, due to the effect of the layer on the distribution of the modal field. 
Application of thicker, lower resistance ITO films will increase waveguide loss and may significantly affect signal detectability; a suitable balance must therefore be achieved between waveguide loss and film resistance in order to optimize the overall sensitivity and electrochemical response of a given device.

The optimum operating wavelength of a sensor device is a further consideration in sensor design. Reduced losses at higher wavelengths for ITO overlayers are to be expected partly because of increasing ITO transmission at longer wavelengths and, in the case of the waveguides considered here, because the field intensity at the superstrate interface is reduced as the mode approaches cutoff, due to the guide asymmetry; this trend is evident in Figures 6 (a) and (b). Some of the increased loss at shorter wavelengths in these spectra may also be due to fiber coupling efficiency variation as the waveguide becomes double-moded; however, none of the overlayered waveguides were observed to be double-moded at $633 \mathrm{~nm}$. A substantial increase in absorption for wavelengths below 500nm may be expected from examination of Figure 5. The reduction in transmission for shorter wavelengths seen in this figure is likely to be due mainly to an increase in the real part of the ITO index for shorter wavelengths, ${ }^{32}$ which increases the reflectivity at the film-air and film-substrate interfaces. On waveguides, the higher ITO film index alters the waveguide parameters so that the field interaction with the film is increased, resulting in a higher absorption.

\section{Conclusions}

The use of transparent electrode coatings to control electrochemical reactions on optical waveguides requires a compromise to be reached between the electrode conductivity and the resultant waveguide attenuation. We have presented a method of fabricating electrode overlayered waveguides using ITO films that demonstrate sufficiently low resistivity for electrochemical sensing applications. The behaviour of the waveguide system at various wavelengths has been considered, and film thickness parameters have been determined that ensure excessive waveguide losses are not introduced. A 1D graded-index waveguide model is sufficient to describe the measured loss characteristics in terms of the absorption of guided modes due to the ITO layers.

The authors are grateful to Harald Gnewuch of the Optoelectronics Research Centre for useful discussions. This work was funded by the EU Environment Programme as part of the BIOPTICAS project. The Optoelectronics Research Centre is an Interdisciplinary Research Centre funded by the UK EPSRC.

\section{References}

1. R.S.Sethi, "Transducer aspects of biosensors" Biosensors and Bioelectronics 9, 243-263 (1994).

2. P.V.Lambeck, "Integrated opto-chemical sensors," Sensors and Actuators B, 8, 103-116 (1992).

3. J.Ingenhoff, B.Drapp and G.Gauglitz, "Biosensors using integrated optical devices," Fresenius J. Anal. Chem. 346, 580-583 (1993).

4. K.Itoh and A.Fujishima, "An application of optical waveguides to electrochemistry: construction of optical waveguide electrodes," J. Phys. Chem. 92, 7043-7045 (1988). 
5. C.Piraud, E.K.Mwarania, J.Yao, K.O.Dwyer, D.J.Schiffrin and J.S.Wilkinson, "Optoelectrochemical transduction on planar optical waveguides," J. Lightwave Tech. 10, 693-699 (1992).

6. J.Kremeskötter, R.Wilson, D.J.Schiffrin, B.J.Luff and J.S.Wilkinson, "Detection of glucose via electrochemiluminescence in a thin-layer cell with a planar optical waveguides," Meas. Sci. Technol. 6, 1325-1328 (1995).

7. J.Kremeskötter, R.Wilson, D.J.Schiffrin, B.J.Luff and J.S.Wilkinson, "Study of luminol electrochemiluminescence with a planar optical waveguide for peroxide sensor application," Electrochemical Society Proceedings 95-1, 1054 (1995).

8. C.R.Lavers, R.D.Harris, S.Hao, J.S.Wilkinson, K.O.Dwyer, M.Brust and D.J.Schiffrin, "Electrochemically controlled waveguide-coupled surface plasmon sensing," J. Electroanalytical Chem. 387, 11-22 (1995).

9. H.Gnewuch and H.Renner, "Mode-independent attenuation in evanescent-field sensors," Appl. Opt. 34, 1473-1483 (1995).

10. W.Huber, R.Barner, Ch.Fattinger, J.Hübscher, H.Koller, F.Müller, D.Schlatter and W.Lukosz, "Direct optical immunosensing (sensitivity and selectivity)," Sensors and Actuators B6, 122-126 (1992).

11. A.N.Sloper, J.K.Deacon and M.T.Flanagan, "A planar indium phosphate monomode waveguide evanescent field immunosensor," Sensors and Actuators B1, 589-591 (1990).

12. Y.Zhou, J.V.Magill, R.M.De La Rue and P.J.R.Laybourn, "Evanescent fluorescence immunoassays performed with a disposable ion-exchanged patterned waveguide," Sensors and Actuators B11, 245--250 (1993).

13. R.Wilson and D.J.Schiffrin, "Use of fluorescamine for the spectrophotometric investigation of primary amines on silanized glass and indium tin oxide-coated glass," Analyst 120, 175-178 (1995).

14. L.Ross, "Integrated optical components in substrate glasses," Glastech. Ber. 62, 285-297 (1989).

15. E.Marantonio, R.E.Zich, I.Montrosset, "Alternative expression of the dispersion equation in multilayered structures,” IEE Proc. J 137, 357-360 (1990).

16. H.W.Gnewuch, private communication.

17. G.L.Yip, J.Albert, "Characterization of planar optical waveguides by $\mathrm{K}^{+}$ion-exchange in glass," Opt. Lett. 10, 151-153 (1985).

18. M.N.Weiss and R.Srivastava, "Determination of ion-exchanged channel waveguide profile parameters by mode index measurements," Applied Optics, 33, pp. 455-458 (1995).

19. A.Miliou, H.Zhenguang, H.C.Cheng, R.Srivastava and R.V.Ramaswamy, 
"Fiber-compatible $\mathrm{K}^{+}-\mathrm{Na}^{+}$ion-exchanged channel waveguides: fabrication and characterization,” IEEE J. Quantum Electron. 25, 1889-1897 (1989).

20. P.S.Chung and M.J.Millington, "Post baking characteristics of single-mode and multimode silver/sodium exchanged waveguides," IEE Proceedings 136, Pt. J, No. 2, 103-107 (1989).

21. Y.Okamura, K.Kitatani and S.Yamamoto, "Low-voltage driving in nematic liquid crystal overlayered waveguide,” J. Lightwave Tech. LT-4, 360-363 (1986).

22. N.Balasubramanian and A.Subrahmanyam, "Electrical and optical properties of reactively evaporated indium tin oxide (ITO) films-dependence on substrate temperature and tin concentration,” J. Phys. D: Appl. Phys. 22, 206-209 (1989).

23. J.L.Yao, S.Hao and J.S.Wilkinson, "Indium tin oxide thin films by sequential evaporation," Thin Solid Films 189, 227-233 (1990).

24. M.Buchanan, J.B.Webb and D.F.Williams, "Preparation of conducting and transparent thin films of tin-doped indium oxide by magnetron sputtering," Appl. Phys. Lett. 37, 213-215 (1980).

25. R.Latz, K.Michael and M.Scherer, "High conducting large area indium tin oxide electrodes for displays prepared by DC magnetron sputtering," Jap. J. Appl. Phys. 30, L149-L151 (1991).

26. T.Maruyama and K.Tabata, "Indium tin oxide thin films prepared by chemical vapour deposition from metal acetates,” Jap. J. Appl. Phys. 29, L355--L357 (1990).

27. M.Scholten and J.E.A.M.van den Meerakker, "On the mechanism of ITO etching: the specificity of halogen acids," J. Electrochem. Soc. 140, 471-475 (1993).

28. M.A.Martinez, J.Herrero and M.T.Gutierrez, "Electrochemical stability of indium tin oxide thin films," Electrochimica Acta 37, 2565-2571 (1992).

29. T.C.Nason, J.A.Moore and T.-M.Lu, "Deposition of amorphous fluoropolymer thin films by thermolysis of Teflon amorphous fluoropolymer," Appl. Phys. Lett. 60, 1866-1868 (1992).

30. J.Kane and H.P.Schweizer, "Chemical vapour deposition of transparent electrically conducting layers of indium oxide doped with tin,” Thin Solid Films 29, 155-163 (1975).

31. J.A.Dobrowolski, F.C.Ho, D.Menagh, R.Simpson and A.Waldorf, "Transparent, conducting indium tin oxide films formed on low or medium temperature substrates by ion-assisted deposition," Appl. Opt. 26, 5204-5210 (1987).

32. J.A.Woollam, W.A.McGahan and B.Johs, "Spectroscopic ellipsometry studies of indium tin oxide and other flat panel display multilayer materials," Thin Solid Films 241, 44-46 (1994). 
33. O.S.Heavens, Optical Properties of Thin Solid Films, (Dover, New York 1965).

34. R.G.Heideman, R.P.H.Kooyman and J.Greve, "Performance of a highly sensitive optical waveguide Mach-Zehnder interferometer immunosensor," Sensors and Actuators B10, 209-217 (1993). 